\title{
A novel full endoscopic annular repair technique combined with autologous conditioned plasma intradiscal injection: a new safe serial therapeutic model for the treatment of lumbar disc herniation
}

\author{
Jizheng Li ${ }^{1 \#}$, Xiaofeng Yuan ${ }^{2 \#}$, Fanbing $\mathrm{Li}^{1}$, Fuke Wang ${ }^{3}$, Yanlin $\mathrm{Li}^{3}$, Enbin Wang ${ }^{4}$, Xianguang Yang ${ }^{3}$, \\ Yaoyu Xiang ${ }^{3}$, En Song \\ ${ }^{1}$ Department of Orthopedics, First Clinical Medical College of Yunnan University of Traditional Chinese Medicine, Kunming, China; ${ }^{2}$ Department \\ of Orthopedics, the First People's Hospital of Kunming, Kunming, China; ${ }^{3}$ Department of Sports Medicine, the First Affiliated Hospital of Kunming \\ Medical University, Kunming, China; ${ }^{4}$ Department of Orthopedics, Baoshan Hospital of Traditional Chinese Medicine, Baoshan, China \\ Contributions: (I) Conception and design: E Song; (II) Administrative support: Y Li, F Wang; (III) Provision of study materials or patients: X Yang, E \\ Wang, F Li; (IV) Collection and assembly of data: J Li, Y Xiang, X Yang; (V) Data analysis and interpretation: E Song, J Li, X Yuan; (VI) Manuscript \\ writing: All authors; (VII) Final approval of manuscript: All authors. \\ \#These authors contributed equally to this work. \\ Correspondence to: En Song. Department of Sports Medicine, the First Affiliated Hospital of Kunming Medical University, No. 295 Xichang Road, \\ Kunming 650031, China. Email: 641634233@qq.com.
}

\begin{abstract}
Backgroundz Recurrent intervertebral disc herniation and an exacerbated degenerative process have been identified as the most important factors contributing to persistent pain and disability after surgical discectomy. Defects in the annulus fibrosus remain a surgical challenge, as the preference for minimally invasive surgical approaches for lumbar microdiscectomy, surgical access, and the specifics of anatomy limit the types of devices that permit annulus fibrosus repair. Furthermore, the intervertebral disc is a relatively avascular structure, and surgical procedures can accelerate the degenerative disc process. This study aimed to evaluate the clinical safety and efficacy of a novel microdiscectomy annular repair technique combined with an autologous conditioned plasma (ACP) intradiscal injection for the treatment of lumbar disc herniation (LDH).
\end{abstract}

Methods: From July 2017 to December 2018 this study recruited 75 patients with LDH (single segment) and randomly divided these patients into the following three groups: group A, full endoscopic discectomy; group B: full endoscopic discectomy and annular repair; group C, full endoscopic discectomy annular repair and ACP intradiscal injection. The pre- and postoperative neurological function and pain status were evaluated by the visual analog scale (VAS) score and the Oswestry disability index (ODI). Patients were followed up once preoperatively, and at 1, 3, and 6 months postoperatively.

Results: The procedure was successfully performed in all cases. No cases required conversion to an open procedure. The preoperative symptoms were alleviated significantly after surgery. The VAS scores for lower back and lower limb pain and ODI score were significantly difference at 1 month, 3 months, 6 months post operation compared to pre-operation scores $(\mathrm{P}<0.05)$. For VAS scores of the lower back, the difference between group $A$ and group $C$ was statistically significant $(A>C)$, as was the difference between group $B$ and group $\mathrm{C}(\mathrm{B}>\mathrm{C})$.

Conclusions: Early results showed that the use of the novel full endoscopic annular repair technique and ACP intradiscal injection serial therapeutic model are beneficial for short term outcomes and demonstrates a reduction in symptomatic disc reherniation with low postoperative complication rates. This new serial therapeutic model may significantly improve the symptoms of postoperative lower back pain. 
Koywords: Full-endoscopy; annular repair; autologous conditioned plasma (ACP); lumbar disc herniation (LDH)

Submitted Oct 16, 2020. Accepted for publication Jan 02, 2021.

doi: 10.21037/apm-20-2257

View this article at: http://dx.doi.org/10.21037/apm-20-2257

\section{Introduction}

Lumbar disc herniation (LDH) is one of the pathological changes related to lumbar disc damage. In terms of treatment, patients who fail in traditional and standardized conservative treatment can elect surgical treatment. Currently, there are two common surgical methods: minimally invasive surgery (MIS) and open surgery. In recent years, MIS has proven superior to open surgery as it involves reduced trauma, less blood loss, marked postoperative benefit, and faster recovery. MIS has thus become the preferred choice of treatment. However, MIS does also entail unavoidable damage to the annulus fibrosus $(1,2)$. Recurrent intervertebral disc herniation and an exacerbated degenerative process have been identified as the most important factors contributing to persistent pain and disability after MIS. Furthermore, inflammatory factors can reach the nerve endings through the annulus fibrosus fissure, which aggravates the onset of lumbar discogenic pain and limits local mobility; this in turn significantly reduces the quality of life of patients with annular disruption. An increasing amount of research has indicated that the maximal repair of the annulus fibrosus is crucial to restoring the normal physiological structure of the intervertebral disc, reshaping the stability of the spine, and improving lumbago conditions (3). However, defects in the annulus fibrosus remain challenging to repair surgically in some ways, such as the preference for MIS approaches in lumbar microdiscectomy, surgical access, and the specific anatomical features involved, limit the types of devices available. This article describes a new safety serial therapeutic model we have developed for the treatment of $\mathrm{LDH}$ - the full endoscopic annular repair technique combined with autologous conditioned plasma (ACP) intradiscal injection-and outlines our surgical experience with this method and its clinical outcomes.

We present the following article in accordance with the CONSORT reporting checklist (available at http://dx.doi. org/10.21037/apm-20-2257).

\section{Methods}

\section{General information}

The inclusive criteria for patients enrolled in this study were the following: (I) an age ranging from 18 to 60 years; (II) diagnosed with LDH in a single segment; (III) failure of conservative treatment for more than 6 weeks; (IV) imaging examinations, including anterio-posterior and lateral film of the lumbar vertebrae, flexion-extension film of the lumbar vertebra, computed tomography (CT) plain scanning of the lumbar vertebrae, and magnetic resonance imaging (MRI) of the lumbar vertebra, that were consistent with one single segment.

The exclusion criteria for patients were the following: (I) presence of lumbar spinal stenosis or severe lumbar disc degeneration; (II) poor general health condition contraindicating surgery, or a previous history of lumbar surgery; (III) lumbar instability or spondylolisthesis; (IV) multisegmented $\mathrm{LDH}$, far lateral $\mathrm{LDH}$, intervertebral foramen $\mathrm{LDH}$, free prolapse $\mathrm{LDH}$, or calcification type LDH; (V) fracture, infection, tumor, or deformity.

From July 2017 to December 2018 this study recruited 75 patients with single-level disc herniation between the L3 and L5 (L3-L4 level, 15 cases; L4-L5 level, 37 cases; L5S1 level, 23 cases), and these patients were and randomly divided into the following three groups (Figure 1): group A, full endoscopic discectomy; group B, full endoscopic discectomy and annular repair; group C, full endoscopic discectomy annular repair and ACP intradiscal injection.

This study was approved by the Ethics Committee of the First Affiliated Hospital of Kunming Medical University (No.2018-L21). All procedures performed in this study involving human participants were in accordance with the Declaration of Helsinki (as revised in 2013). Informed consent was taken from all the patients.

\section{Materials}

The endoscope used for the procedure and the annular 

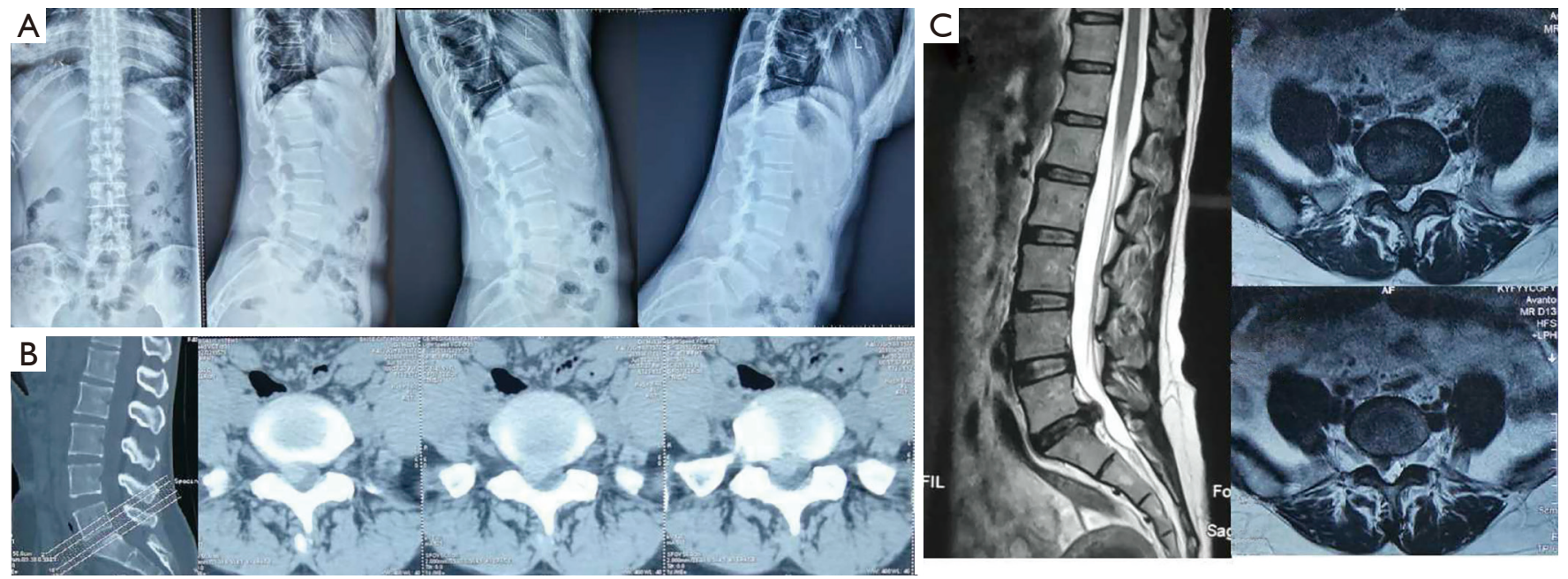

Figure 1 A 40-year-old female patient. (A) Preoperative lumbar radiological images revealing no dynamic instability. (B) A preoperative CT scan revealing an L5-S1 level lumbar disc herniation with no calcification. (C) Preoperative MRI images revealing an L5-S1 level lumbar disc herniation. MRI, magnetic resonance imaging.

repair device (Figure 2) was produced by 2020 Medical Technology Co., Ltd. (Beijing, China). The Arthrex ACP TM Double Syringe System used was produced by Arthrex (Naples, FL, USA) (Figures 3 and 4).

\section{Surgical procedure}

\section{Full endoscopic discectomy (group A)}

Surgery was performed in a prone position under local anesthesia. C-arm fluoroscopy was applied to locate the operative segments. At L3-L4 level and L4-L5 level, a transforaminal endoscopic lumbar discectomy technique was used. The skin entry point for needle insertion was calculated based on pre-operative MRI and CT scan by measuring the distance from the midline, and the needle trajectory was aimed to target the ruptured fragment. The guiding wire was introduced into the foramen; foraminotomy was then performed with different reamer types to remove the partial superior articular process. The working cannula and spine endoscope were introduced to the L5-S1 level using an interlaminar endoscopic lumbar discectomy technique. The entry point was made in reference to the medial pedicular line in the AP view and the spino-laminar line on the lateral view with direction of the needle towards the superior endplate of the S1 vertebra. Once the needle reached the spino-laminar junction, the needle tract was dilated with a series of dilators, and the endoscope was introduced. The ligamentum flavum was split to expose the nerve root and herniated region. The herniated disc tissue was removed by forceps. After the nerve root decompression was completed, the incision was closed in routine fashion.

\section{Full endoscopic discectomy and annular repair group (group B)}

The full endoscopic discectomy procedure was performed in the same fashion as that described above for group A. The annular tear segment was smoothed by radiofrequency. Once the tear was prepared for suturing, the interval between the tear and nerve root was identified, and the nerve root was retracted and protected by the working cannula. The novel Disposable Annular Stapler (2020 Medical Technology Co., Ltd., Beijing, China), an automatic stitching and tying device, was used to automatically tie the first surgical knot. Then, a curved puncture needle was used to pierce through one side of the annular tear. The knob on the stapler handle was then turned forward until the arrow on the handle was aligned with the yellow marker line on the knob. The triangle button was pushed forward to the front end. The knob was then turned backward until the arrow on the handle was aligned with the red marker line on the knob, the annular stapler was then pulled out, and the knot slid in and locked automatically. Two more knots were tied, and the knot pusher was used from outside to inside. The suture was cut by a suture cutter under endoscopic monitoring (Figure 2). After the suture was completed, the 

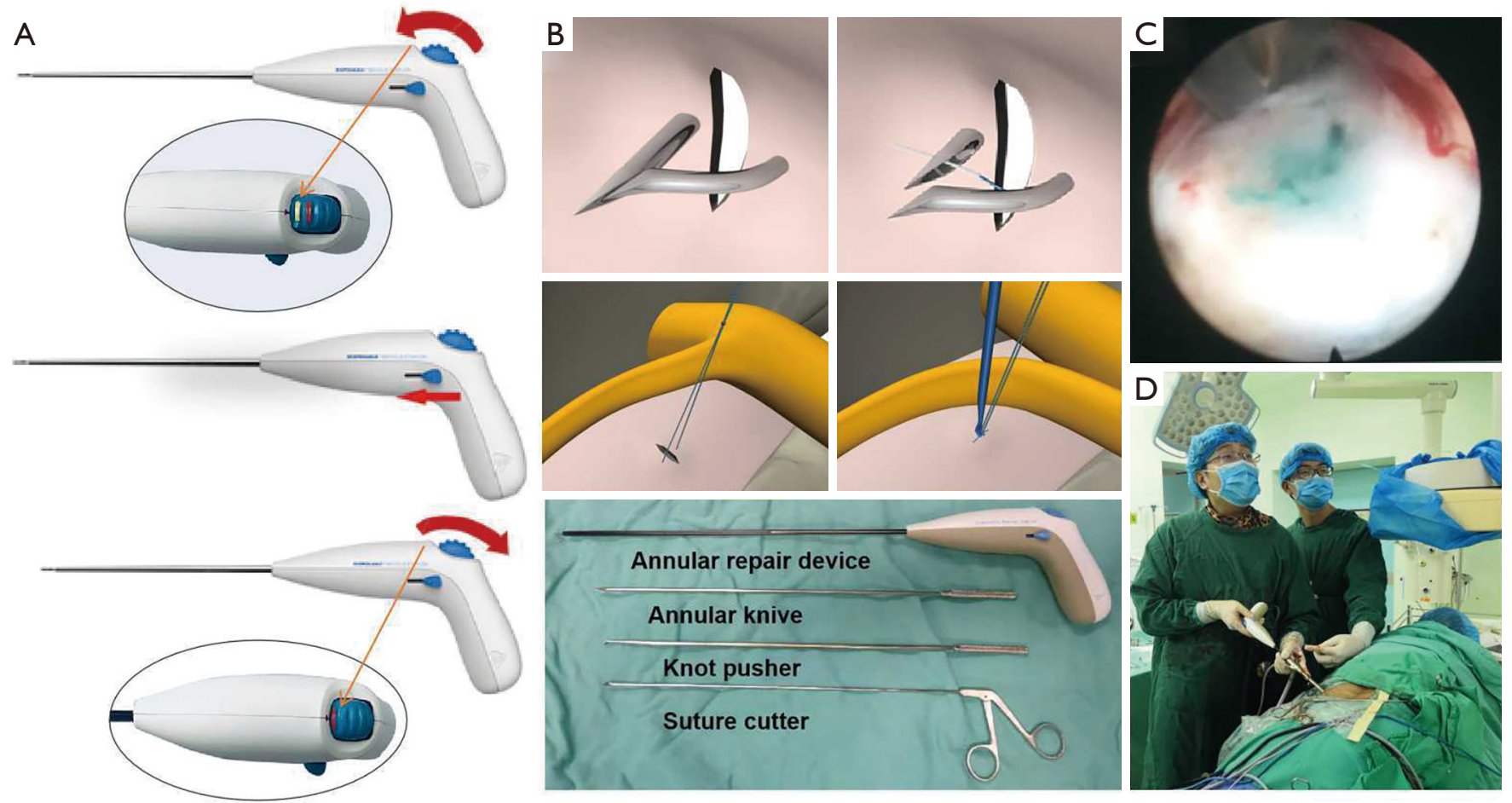

Figure 2 Annular repair procedure. (A,B) The novel Disposable Annular Stapler (2020 Medical Technology Co., Ltd., Beijing, China) is an automatic stitching and tying device which can automatically tie the first surgical knot. A curved puncture needle pierces through one side of the tear, and then two more knots are tied from outside to inside. (C,D) An intraoperative image showing annular tear closure.

incision was closed in routine fashion.

\section{Full endoscopic discectomy annular repair and ACP intradiscal injection (group C)}

Full endoscopic discectomy and annular repair was performed in the same fashion as described for group B above. At 1 week, 1 month, and 2 months after the discectomy procedure, patients underwent an ACP intradiscal injection procedure. A volume of $15 \mathrm{~mL}$ of blood was drawn into the double syringe system (Arthrex, Naples, FL, USA) for a single spin in a centrifuge (Hettich ROTOFIX 32 A, Arthrex, Naples, FL, USA) at 1,500 r/mim for 5 minutes. The ACP was procured by pulling back on the smaller syringe to remove the yellow leukocytepoor platelet-rich plasma (PRP) layer, leaving the lower leukocyte-rich red blood cell pack behind. The volume of available PRP produced during the ACP procedure differed for each individual, ranging from 4 to $5.8 \mathrm{~mL}$ (Figure 3). Surgery was performed with patients in a prone position under local anesthesia. The intervertebral disc segments were located by a C-arm during the operation. An 18-gauge spinal needle was inserted into the central area of the intervertebral disc, and 1.2-2.5 $\mathrm{mL}$ of ACP was injected into the intervertebral disc, as shown in Figure 4.

The patients were mobilized 5 hours after surgery. A flexible back brace was used for 3 weeks. After leaving the hospital, patients were encouraged to resume their daily routine and were followed up as outpatients at the hospital ward.

\section{Evaluation index}

To assess lower back pain and lower limb pain, visual analog scale (VAS) and Oswestry disability index (ODI) scores in group A and B were evaluated before operation, and at 1 month, 3 months, and 6 months after the operation. Meanwhile, the VAS and ODI scores in group $\mathrm{C}$ were evaluated at 1 month, 3 months, and 6 months after the last ACP injection, so as to evaluate the therapeutic effect and technical value of the novel full endoscopic annular repair technique and ACP intradiscal injection serial therapeutic model. 

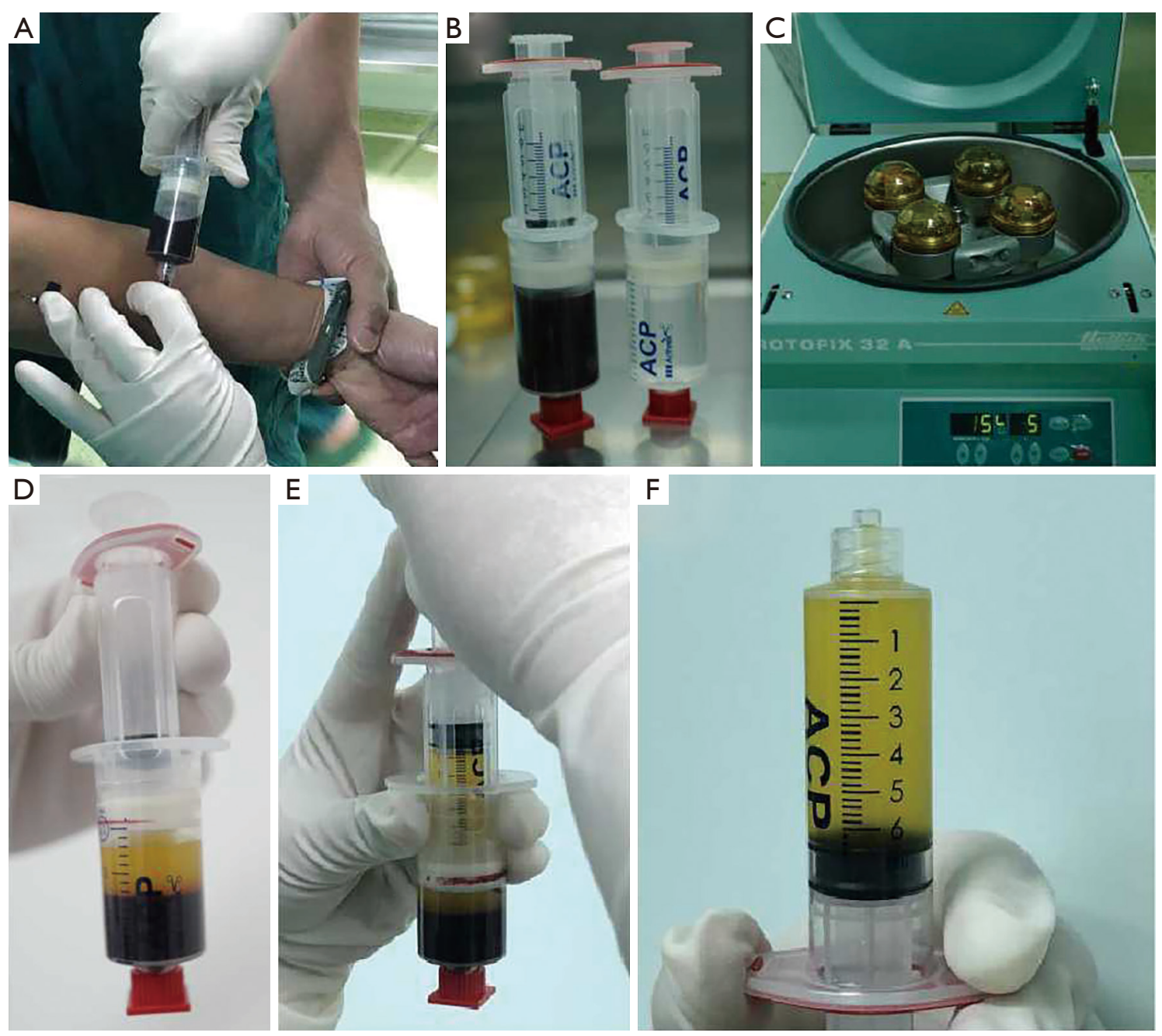

Figure 3 Preparation of the ACP. (A) Fifteen milliliters of the patient's peripheral blood are drawn into a double syringe system (Arthrex Inc., Naples, FL, USA). (B) The normal saline weighing and balancing method. (C) The entire syringe containing the whole blood is centrifuged at 1,500 rpm for 5 minutes. (D) The syringe components with blood are separated into the leukocyte-rich red blood cell pack and the final injection syringe containing the ACP. (E) The smaller syringe is unscrewed from within the larger syringe. (F) The ACP is carefully drawn into the smaller syringe. ACP, autologous conditioned plasma.

\section{Data analysis}

Clinical outcomes were assessed by the visual analogue scale (VAS; $0-10$, with $0=$ no pain), and functional outcomes were scored with the ODI (ODI; 0-100\%). SPSS v.22.0 (SPSS Inc., Chicago, IL, USA) was used for statistical analysis, and the measurement data are expressed as mean \pm standard deviation. One-way analysis of variance (ANOVA) was adopted in the intergroup measurement data comparison, while repeated-measure ANOVA was used in the intragroup measurement data comparison. The least significant difference (LSD) method was employed for multiple comparisons, which are expressed as mean \pm standard deviation. Fisher's exact test was use for comparing enumeration data. A P value $<0.05$ indicated the differences were statistically significant.

\section{Results}

Comparison of VAS scores of lower back pain before and after operation in each group (Table 1) revealed no significant differences among the three groups before operation, 1 month after operation, and 3 months after 

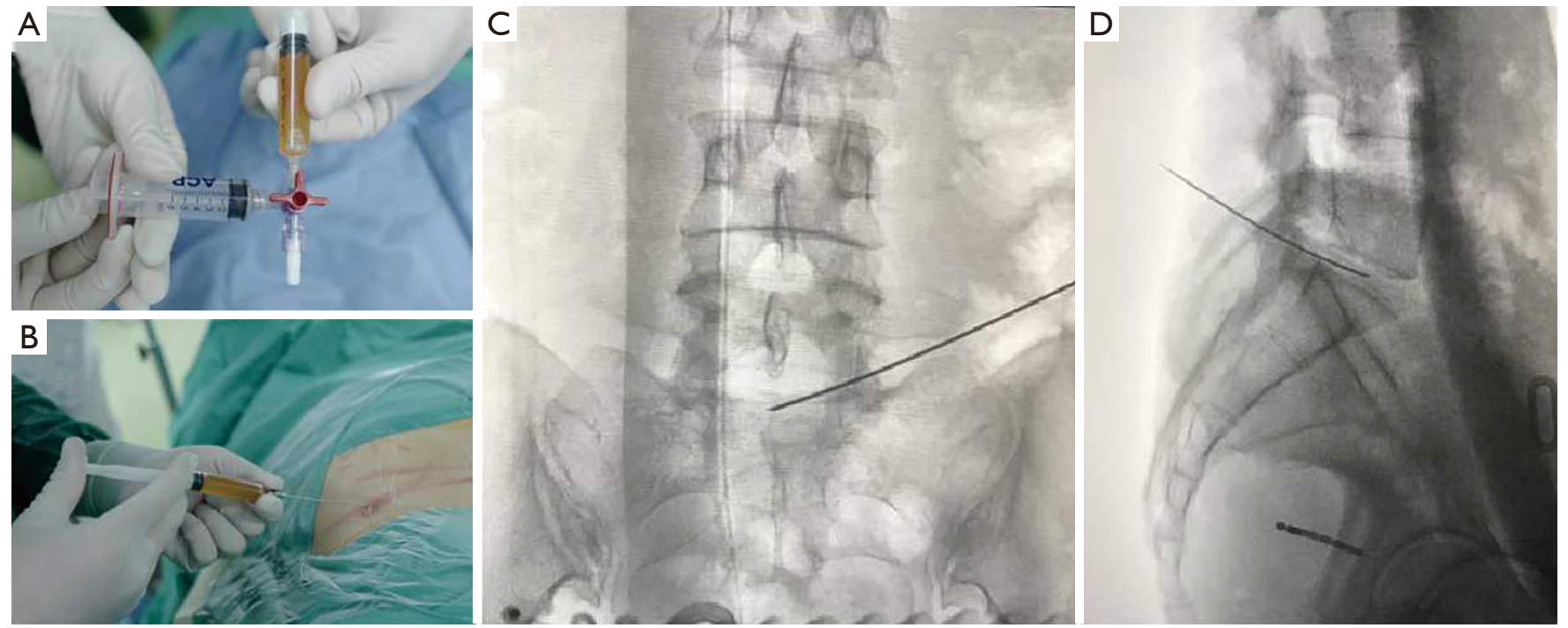

Figure 4 ACP intradiscal injection. (A) ACP is transferred into a $5 \mathrm{~mL}$ syringe by a T-connector. (B) Intra-operative ACP intradiscal injection. (C) Antero-posterior view of the intra-operative ACP intradiscal injection. (D) Lateral view of the intra-operative ACP intradiscal injection. ACP, autologous conditioned plasma.

Table 1 Comparison of VAS scores of lower back pain before and after operation in each group $(\bar{x} \pm \mathrm{s})$

\begin{tabular}{|c|c|c|c|c|c|c|}
\hline Group & $\mathrm{n}$ & \multicolumn{4}{|c|}{ Low back pain VAS } & Statistics \\
\hline B & 25 & $7.88 \pm 0.19$ & $3.36 \pm 0.19$ & $2.12 \pm 0.11$ & $1.80 \pm 0.12$ & $F=320.623, P<0.001$ \\
\hline
\end{tabular}

VAS, visual analog scale.

operation. There was a significant difference 6 months after operation: the difference between the group $\mathrm{A}$ and group $C$ scores was statistically significant $(A>C)$, as was that between the group $B$ and group $C$ scores $(B>C)$. There was no significant difference between group $A$ and group $B$ 3 months after operation or 6 months after operation. The difference at other time points was statistically significant. The score difference across time points for group C was statistically significant: as time progressed, the VAS score showed a downward trend.

Comparison of VAS scores of the lower limbs before and after operation in each group (Table 2), also revealed no significant differences among the three groups before operation, 1 month after operation, and 3 months after operation. There was significant differences 6 months after operation: the difference between group $\mathrm{A}$ and group $\mathrm{C}$ was statistically significant $(A>C)$, as was that between group $A$, group $B$, and group $C$ at different time points. As time progressed, the VAS score showed a downward trend.

In the comparison of ODI scores for patients in each group (Table 3), there was no significant difference in oneway ANOVA at any time point $(\mathrm{P}>0.05)$. However, the repeated measure ANOVA analysis for ODI score found significant differences $(\mathrm{P}<0.05)$ among the three groups for ODI score before operation, and at 1 month, 3 months, and 6 months after the operation. Multiple comparisons with the LSD method revealed that the ODI score increased as time progressed in all groups.

There was 1 case of postoperative recurrence in group A, yielding a recurrence rate of $4.0 \%$; there was no post- 
Table 2 Comparison of VAS scores of the lower limbs before and after operation in each group $(\bar{x} \pm s)$

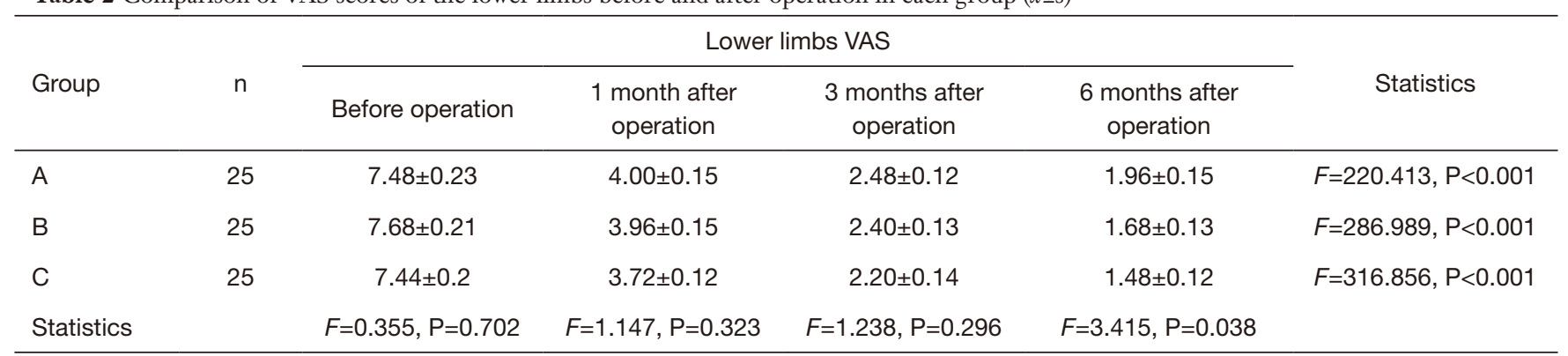

VAS, visual analog scale.

Table 3 Comparison of ODI score for patients in the different groups $(\bar{x} \pm \mathrm{s})$

\begin{tabular}{|c|c|c|c|c|c|c|c|}
\hline Group & $\mathrm{N}$ & Before operation & $\begin{array}{c}1 \text { month after } \\
\text { operation }\end{array}$ & $\begin{array}{c}3 \text { months after } \\
\text { operation }\end{array}$ & $\begin{array}{c}6 \text { months after } \\
\text { operation }\end{array}$ & $\mathrm{F}$ & $P$ \\
\hline A & 25 & $74.18 \pm 9.92^{d}$ & $40.38 \pm 5.74^{c}$ & $25.38 \pm 6.89^{b}$ & $18.65 \pm 12.49^{a}$ & 385.065 & 0.000 \\
\hline C & 25 & $76.57 \pm 14.59^{d}$ & $36.15 \pm 9.07^{c}$ & $22.66 \pm 7.76^{b}$ & $14.33 \pm 4.63^{\mathrm{a}}$ & 288.178 & 0.000 \\
\hline $\mathrm{F}$ & & 0.250 & 1.765 & 1.935 & 2.190 & & \\
\hline
\end{tabular}

${ }^{a},{ }^{b},{ }^{c}$, and ${ }^{d}$ refer to the results of intragroup comparisons; different letters indicate that the differences of intragroup comparison were statistically significant $(\mathrm{P}<0.05)$. ODI, Oswestry disability index.

operative recurrence cases in group B or group C, both of which had a recurrence rate of $0.0 \%$. The analysis of the recurrence rate of the patients in the three groups using Fisher's exact test revealed no statistically significant differences $(\mathrm{P}=1.000>0.05)$.

\section{Discussion}

\section{Anatomical and physiological characteristics of annulus fibrosus}

The annulus fibrosus is a rough-textured tissue surrounding the nucleus pulposus of the intervertebral disc which crisscrosses between the upper and lower vertebrae. Together with the anterior and posterior longitudinal ligaments, the annulus fibrosus plays an important role in connecting vertebral bodies above and below, limiting nucleus pulposus, absorbing concussion, controlling the range of motion, and stabilizing the spine. Lumber discectomy is currently recognized as the gold standard for surgical treatment of $\mathrm{LDH}$, but surgical treatment inevitably causes damage to the annulus fibrosus $(4,5)$. Reticular degeneration and hyaline degeneration caused by physiological degeneration or pathological changes are also important causes of annulus fibrosus injury. Due factors related to the blood supply, the self-repair ability of annulus fibrosus is weak. When it is damaged but not effectively repaired, and the annular closed space is not restored in time, intervertebral disc herniation can recur and the process of intervertebral disc tissue degeneration is accelerated (6). Some researchers hold the view that annulus fibrosus defects larger than $6 \mathrm{~mm}$ can increase the recurrence rate of intervertebral disc herniation by an average of $15 \%$, with the maximum reported increase being as high as $27 \%(7)$. Therefore, conducting surgical intervention on the annulus fibrosus with a defect larger than $6 \mathrm{~mm}$ is necessary, and this need has given rise to the development of annular repair technology.

\section{Significance of annular repair}

Clinical studies have shown that annular repair technology can not only significantly reduce the recurrence rate after nucleus pulposus excision, but can also reduce postoperative pain, scar formation, and nerve adhesion. The application 
of annulus fibrosus mechanical suture technology has the following advantages: (I) it can establish a mechanical barrier to prevent the recurrence of disc herniation. (II) The amount of nucleus pulposus removed during nucleus pulposus removal can be affected by subjective factors of the operator, which often leads to excessive removal of nucleus pulposus, leaving the intervertebral space further collapsed and degenerated. (III) Annulus fibrosus suture repair forms a closed space inside the intervertebral disc to prevent the leakage of various biological agents and provides the volume basis for the biological treatment of intervertebral disc.

At present, many kinds annulus fibrosus suture and plugging technology have been widely used in clinical practice and have achieved good results, while endoscopic lumbar annulus fibrosus repair has been gradually promoted (8). However, in a study involving 478 patients, 137 patients could not be sutured directly due to poor tissue conditions of the annulus fibrosus, excessive protrusion range, starshaped tear, complex anatomy, and other reasons (9). It can be seen that simple circumferential suture cannot effectively prevent the degradation process, and the recurrence probability is still very high (10).

\section{Other treatments for annulus fibrosus injury}

Due to the different degrees of intervertebral disc degeneration, the current repair methods for annulus fibrosus have been extended using gene tissue engineering, biological therapy, implantation biomaterials, and other technologies (11). Biological therapy includes stem cell therapy, growth factor and/or cytokine injection, gene therapy, etc. The purpose of biological therapy is to promote the proliferation and differentiation of intrinsic stem cells and/or progenitor cells of intervertebral disc by various means, to correct the environmental disorder in the early degenerative intervertebral disc, and to promote the synthesis and metabolism of extracellular matrix (12). At present, tissue engineering therapy research has made clear progress in the construction of intervertebral disc scaffold, matrix material research and development, seed cell selection, and other areas. Another approach, growth factor injection therapy, is aimed at directly injecting growth factors that promote cell proliferation, migration, and differentiation into intervertebral disc tissue, so as to alleviate the progress of intervertebral disc degeneration at the molecular, cell, and histomorphology level, which promotes the repair of ruptured intervertebral discs (13). An example of this therapy is using alpha granules in PRP to release platelet-derived growth factor (PDGF) and other growth factors after platelet activation (14).

\section{The function of PRP}

After platelet activation, PRP releases many growth factors and forms platelet gel. The bioactive molecules in the gel stimulate the proliferation of fibroblasts, smooth muscle cells, and osteoblasts, and promote the repair of various injuries. At present, PRP has been widely used in orthopedics, stomatology, neurosurgery, pain reduction, rehabilitation medicine, and other departments. In sports medicine, the treatment of acute muscle strain, tendon disease, myofascial injury, osteoarthropathy, and other sports trauma diseases is inseparable from PRP (15-17). Alsousou et al. (18) have reported that PRP can directly stimulate bone marrow stromal cells to differentiate into intervertebral disc cells, and promote the synthesis of two main components of the extracellular matrix: aggrecan-1 (Agc1) and collagen. Aggrecan can promote the absorption of water and hydration of the intervertebral disc, while collagen can anchor soft tissue on bone tissue, which induces an antistretching effect. Therefore, PRP can restore the hydrostatic pressure of the intervertebral disc, improve its load, and relieve discogenic lower back pain to a certain extent. ACP made by Arthrex is also a kind of PRP. What makes the ACP system so attractive is that it lacks a second centrifugation step but still provides a platelet concentrate solution in an easily removable syringe for application.

\section{Significance of annular repair combined with ACP in the treatment of $\mathrm{LDH}$}

After lumber discectomy for LDH, the internal structures of the intervertebral disc such as the nucleus pulposus tissue, endplate tissue, inner layer, and outer annulus fibrosus tissue are damaged to varying degrees, resulting in a significant increase in postoperative recurrence, usually at the original incision or rupture (19). In vivo and in vitro model studies of PRP injection technology have found that it can promote nucleus pulposus regeneration, increase the expression of the chondrogenic differentiation gene, and increase the level of the extracellular matrix. After the first stage of annulus fibrosus suture, ACP was injected into the intervertebral disc in the second stage to promote biological regeneration and repair. PRP is significant for a number of reasons. (I) A large number of growth factors in PRP can promote the synthesis of collagen and aggrecan in 
the intervertebral disc, improve the water absorption and hydration of intervertebral disc after annulus fibrosus suture, and improve the repair effect of annulus fibrosus. (II) After annular repair, PRP injection can downregulate a variety of inflammatory factors, such as nitric oxide, interleukin-1, matrix metalloproteinases, prostaglandin E2, and tumor necrosis factor- $\alpha$, and improve the inflammatory response induced by annulus fibrosus repair and LDH (20). (III) PRP can induce stem cells in nucleus pulposus to differentiate into nucleus pulposus cells, promote the healing of annulus fibrosus suture repair, and delay the degeneration of intervertebral disc (12). Indeed, it was found that the improvement of intervertebral disc degeneration and the VAS score of lower back pain after combined treatment was better than that obtained from standard treatment.

\section{Our surgical experiences and modifications}

When using the annular stapler the puncture direction must be parallel to the intervertebral space, and not angled toward the endplate, as this can cause stitching failure and cause endplate damage. If the blood extraction fails to reach $15 \mathrm{~mL}$ (due to the patient's blood viscosity, the influence of air in the syringe, etc.), the normal saline weighing and balancing method is adopted to reduce the centrifugal error. In our practice, ACP is transferred into a $5 \mathrm{~mL}$ syringe by a T-connector before the intradiscal injection procedure is conducted.

\section{The innovation and advantages of this serial therapeutic model}

Using the full endoscopic discectomy procedure can maximally preserve the normal intervertebral disc tissue. Furthermore, applying the novel annular repair technique to close the annular tear-a technique we call "mechanical annular repair" - can create an enclosed space for the disc tissue repair. We thus consider ACP intradiscal injection a type of biological therapy that can promote disc tissue regeneration and extracellular matrix synthesis. This new serial therapeutic model for the treatment of $\mathrm{LDH}$ is minimally invasive and precise, and provides effective repair and regeneration.

In conclusion, our findings indicate that this new serial therapeutic model can significantly reduce the recurrence rate of disc herniation, reduce postoperative intraspinal fibrotic scar formation, reduce the release of inflammatory mediators from the nucleus pulposus, promote disc regeneration and extracellular matrix synthesis, maximally preserve intervertebral disc function, reduce postoperative back pain, and improve postoperative quality of life.

\section{Acknowledgments}

Funding: This study was supported by Yunnan Province Clinical Center for Bone and joint Diseases Programme (ZX2019-03-04).

\section{Footnote}

Reporting Checklist: The authors have completed the CONSORT reporting checklist. Available at http://dx.doi. org/10.21037/apm-20-2257

Data Sharing Statement: Available at http://dx.doi. org/10.21037/apm-20-2257

Conflicts of Interest: All authors have completed the ICMJE uniform disclosure form (available at http://dx.doi. org/10.21037/apm-20-2257). The authors have no conflicts of interest to declare.

Ethical Statement: The authors are accountable for all aspects of the work in ensuring that questions related to the accuracy or integrity of any part of the work are appropriately investigated and resolved. This study was approved by the Ethics Committee of the First Affiliated Hospital of Kunming Medical University (No.2018-L21). All procedures performed in this study involving human participants were in accordance with the Declaration of Helsinki (as revised in 2013). Informed consent was taken from all the patients.

Open Access Statement: This is an Open Access article distributed in accordance with the Creative Commons Attribution-NonCommercial-NoDerivs 4.0 International License (CC BY-NC-ND 4.0), which permits the noncommercial replication and distribution of the article with the strict proviso that no changes or edits are made and the original work is properly cited (including links to both the formal publication through the relevant DOI and the license). See: https://creativecommons.org/licenses/by-nc-nd/4.0/.

\section{References}

1. Ambrossi GL, McGirt MJ, Sciubba DM, et al. Recurrent 
lumbar disc herniation after single-level lumbar discectomy: incidence and health care cost analysis. Neurosurgery 2009;65:574-8; discussion 578.

2. Ahn Y. Current techniques of endoscopic decompression in spine surgery. Ann Transl Med 2019;7:S169.

3. Sloan SR Jr, Lintz M, Hussain I, et al. Biologic Annulus Fibrosus Repair: A Review of Preclinical In Vivo Investigations. Tissue Eng Part B Rev 2018;24:179-90.

4. Bowles RD, Setton LA. Biomaterials for intervertebral disc regeneration and repair. Biomaterials 2017;129:54-67.

5. Li X, Dou Q, Kong Q. Repair and Regenerative Therapies of the Annulus Fibrosus of the Intervertebral Disc. J Coll Physicians Surg Pak 2016;26:138-44.

6. Chu G, Shi C, Wang H, et al. Strategies for Annulus Fibrosus Regeneration: From Biological Therapies to Tissue Engineering. Front Bioeng Biotechnol 2018;6:90.

7. Long RG, Ferguson SJ, Benneker LM, et al. Morphological and biomechanical effects of annulus fibrosus injury and repair in an ovine cervical model. JOR Spine 2019;3:e1074.

8. Li ZZ, Cao Z, Zhao HL, et al. Analysis of the technical key points and clinical effect of full-endoscopic lumbar annulus fibrosus suture. Zhongguo Gu Shang 2020;33:498-504.

9. Blanquer SB, Sharifi S, Grijpma DW. Development of poly(trimethylene carbonate) network implants for annulus fibrosus tissue engineering. J Appl Biomater Funct Mater 2012;10:177-84.

10. Suh BG, Uh JH, Park SH, et al. Repair using conventional implant for ruptured annulus fibrosus after lumbar discectomy: surgical technique and case series. Asian Spine J 2015;9:14-21.

11. Adams MA, Roughley PJ. What is intervertebral disc degeneration, and what causes it? Spine (Phila Pa 1976) 2006;31:2151-61.

Cite this article as: Li J, Yuan X, Li F, Wang F, Li Y, Wang E, Yang X, Xiang Y, Song E. A novel full endoscopic annular repair technique combined with autologous conditioned plasma intradiscal injection: a new safe serial therapeutic model for the treatment of lumbar disc herniation. Ann Palliat Med 2021;10(1):292-301. doi: 10.21037/apm-20-2257
12. Tuakli-Wosornu YA, Terry A, Boachie-Adjei K, et al. Lumbar intradiskal Platelet-Rich Plasma (PRP) injections: a prospective, double-blind, randomized controlled study. PM R 2016;8:1-10.

13. Xie X, Zhang C, Tuan RS. Biology of platelet-rich plasma and its clinical application in cartilage repair. Arthritis Res Ther 2014;16:204.

14. Mussano F, Genova T, Munaron L, et al. Cytokine, chemokine, and growth factor profile of platelet-rich plasma. Platelets 2016;27:467-71.

15. Hou Y, Shi G, Shi J, et al. Study design: in vitro and in vivo assessment of bone morphogenic protein 2 combined with platelet-rich plasma on treatment of disc degeneration. Int Orthop 2016;40:1143-55.

16. Xu HH, Li SM, Fang L, et al. Platelet-rich plasma promotes bone formation, restrains adipogenesis and accelerates vascularization to relieve steroids-induced osteonecrosis of the femoral head. Platelets 2020. [Epub ahead of print]. doi:10.1080/09537104.2020.1810221.

17. Pirvu TN, Schroeder JE, Peroglio M, et al. Platelet-rich plasma induces annulus fibrosus cell proliferation and matrix production. Eur Spine J 2014;23:745-53.

18. Alsousou J, Ali A, Willett K, et al. The role of platelet-rich plasma in tissue regeneration. Platelets 2013;24:173-82.

19. Lebow RL, Adogwa O, Parker SL, et al. Asymptomatic same-site recurrent disc herniation after lumbar discectomy: results of a prospective longitudinal study with 2-year serial imaging. Spine (Phila Pa 1976) 2011;36:2147-51.

20. Gui K, Ren W, Yu Y, et al. Inhibitory effects of plateletrich plasma on intervertebral disc degeneration: a preclinical study in a rabbit model. Med Sci Monit 2015;21:1368-75.

(English Language Editor: J. Gray) 